\title{
Research on Present Situation and Development Strategy of HOPSCA in Linyi City
}

\author{
Zhongxiu Liu \\ School of Civil Engineering and Architecture line \\ Linyi University \\ Linyi, Shandong, China
}

\begin{abstract}
Based on field investigation, expert interview and data collection, this paper analyzes the development status of HOPSCA in Linyi city, and summarizes the characteristics of HOPSCA in Linyi: late starting and rapid development; mainly concentrated in Lanshan District, basically according to the distribution of traditional business circle; the core function is business functions. Then, from the four aspects of location, position, planning and design, and investment operation, the paper concludes the strategy suitable for the development of HOPSCA in Linyi city.
\end{abstract} city

Keywords-HOPSCA; feature; development; strategy; Linyi

\section{INTRODUCTION}

The HOPSCA is a at least three-level city living space in which hotels, offices, parks, shopping malls, clubs, and apartments are located. Service functions, and establish a multi-functional and high-efficiency complex, which is a complex space with high concentration of various property forms.

The HOPSCA is a hot issue in the development of Linyi city. Because the HOPSCA means intensive land-use, high value, large volume, representing the highest level of urban development, the product of urbanization in a certain heigh.

\section{DEVELOPMENT STATUS AND CHARACTERISTICS OF HOPSCA IN LINYI CITY}

\section{A. Development Status and Development Trend of HOPSCA in Linyi City}

At present, Linyi city center has been built three HOPSCA: Jiulong - Oscar, Harmony Square, Wanyue City. In 2014, the city's key projects include China City Plaza, China Resources Center, Lu Business Center, Qilu Park Plaza, Red Star International Plaza, etc., of which China Resources Center, Lu Center, Qilu Park Plaza has started selling. Since 2015, Dalian Wanda Group plans to build Wanda Plaza in Beicheng New District, Luozhuang District and Hedong District. 2013-2020 is expected to add 11 million square meters of HOPSCA; 2013-2016, 18 new HOPSCA; 2017-2020, 16 new HOPSCA. It can be said that the future of Linyi HOPSCA will face greater room for development.

\section{B. Characteristics of Linyi HOPSCA}

First, from the development process, the late starting and the rapid development momentum.

China's earliest HOPSCA in 1980s, mainly in shopping and entertainment functions, such as Guangzhou Tianhe City. In the 1990s, HOPSCA construction speed. After entering the 21st century, the process of urbanization is accelerating, the land and resources of the city are becoming scarce, and the function of city center is becoming more and more intensive. HOPSCA function is more and more, such as Shenzhen China Resources Center, Ningbo Wanda Plaza. They have the elements of modern HOPSCA from the functional format, planning and design, environmental landscape, internal and external transportation. [1]

The first true HOPSCA was Jiulong Oscar in Linyi City, built in 2007, opened in 2013. It Located in the north of People's Square, belong to the core business district. A total investment is more than 1.5 billion, construction area of 430,000 square meters. The project is positioned as a multifunctional $\mathrm{CBD}$, including $5 \mathrm{~A}$ super high-rise office buildings and large department stores, including business, office, catering, leisure, entertainment and residence. Next to it is another HOPSCA - Wanyue City, located on the west of the People's Square, with Linyi City Library, 5A office buildings, hotel apartments, noble residential, cultural and business centers and other diversified formats. The total construction area about 230,000 square meters, the highest about 150 meters, the first height for the city center. Currently operating another HOPSCA is a Harmonious Square, which is located in the junction of Tongda Road and Jiefang Road in Lanshan District, located in the Western Suburbs Shopping District, investment 2.2 billion, an area of 140,000 square meters, the introduction of more than 500 international brands, type of business including catering, entertainment, leisure, culture, sports.

After 2013, Linyi HOPSCA development entered the fast lane, a variety of planning projects have emerged. Linyi HOPSCA also develop rapidly with the Linyi city economic development and the level of urbanization. China Resources Group, Lushang Group, Wanda Group and other famous groups have settled in Linyi real estate market to develop HOPSCA. 
Second, from the overall spatial layout, the recent Linyi HOPSCA mainly concentrated in the Lanshan District, Beicheng New District, Luozhuang District and Hedong District has just started. The location of HOPSCA is also consistent with the distribution of the traditional business district in Linyi City, such as Jiulong - Oscar, China Resources Center and Wanyue City distribution in the People's Square-- the core business district; Harmony Square, Wanli Square and China City Plaza is located in the western suburbs--the traditional business district. Due to the short construction time and the low occupancy rate, Beicheng New District is in the process of being formed. LuShang Center and Qilu Garden Square, Ego Street, Hongxing International Plaza and other projects are becoming the new business district of Beicheng New District. the planning and construction projects in Luozhuang District and Hedong District are also in the region in the traditional business district. In general, the layout of the HOPSCA in Linyi City shows a relatively concentrated, consistent with the characteristics of traditional shopping district.

Thirdly, from the view of internal function matching, according to the analysis of the proportion of the interior area of Linyi which has been built and under construction, the dominant function is business, that is, the combination of commercial core is Linyi HOPSCA project mainstream. Follow-up ranking were residential, business, cultural and entertainment. According to international experience, the trend of HOPSCA development will be more emphasis on the use of culture in the project and the construction of public space, and only to achieve the appropriate functional format match to meet the original intention of HOPSCA construction, Economic, environmental and social benefits. Therefore, the commercial function of "one industry dominance" of the situation to be improved in the future.

\section{THE DEVELOPMENT STRATEGY OF HOPSCA IN LINYI CITY}

HOPSCA is to meet the needs of urban development and reconstruction, so the project development strategy must fit the city orientation, urban structure and development trends and other government planning and macro needs; Project development model and direction, to develop a comprehensive urban complex development strategy for the development process steps to strategic guidance and control.

From the development process of HOPSCA, it is necessary to form a unified development strategy based on the four aspects of project location, positioning, design and investment management, in order to effectively guide and control the whole development process.

\section{A. Location}

For the HOPSCA such a large investment, long cycle, large volume, functional complex, obvious driven function, the large social impact projects, location is particularly important. There are many factors influencing the location of the HOPSCA, including natural factors, economic factors, social development factors, political factors, historical and cultural factors and so on. Through the field investigation, the data are collected and analyzed, and some mathematical methods, such as Fuzzy Analytic Hierarchy Process (AHP), are used to analyze and select the optimum location.

From the development of HOPSCA can be seen, the initial HOPSCA is often located in the city core area, the core business district, CBD and other important positions. Then gradually outward expansion, development to the city center area, vice city center, city new district, suburban junction, city characteristic industry gathering area and so on Linyi HOPSCA construction also embodies this law. The earliest commercial Jiulong • Oscar is in the city CBD's core location, and Harmony Square is in the traditional western suburbs. Other HOPSCA under construction and planning are also at the core of the region.

At present, the development of HOPSCA in Linyi has entered a stage of rapid development, mainly to the development of real estate developers. And they first consider the economic benefits of the site, often neglect the environmental and social benefits. In the urban area of functional allocation, coordinated development of regional economy consider less. In view of this, Linyi City from the beginning of 2014, has started the "Linyi City HOPSCA Development Plan" preparation organization and implementation work.

\section{B. Positioning}

The second element of the HOPSCA development strategy is the formation of precise market positioning. It Can be divided into enterprise positioning, brand image positioning, functional positioning, scale positioning, format positioning, customer positioning, business strategy positioning and so on.

At present, the HOPSCA in Linyi is basically the introduction of well-known real estate enterprises to carry out construction. Linyi City and Dalian Wanda Group, Evergrande Real Estate, China Resources Group, LuShang Group, Shanghai Meikailong, Vanke Group and Dalian Friendship Group and other well-known real estate development companies directly docking, HOPSCA project as soon as possible. These well-known corporate with obvious brand effect, the high-end corporate image, rich experience in the development of HOPSCA, is bound to develop a positive role in promoting Linyi HOPSCA.

Linyi HOPSCA in the regional function of positioning, in the old city area, to improve the traffic as the focus, to create a number of urban integrated functional areas, to effectively break down the flow of people, logistics, traffic and so on. In Beicheng New District, we should focus on catering, leisure, entertainment, shopping and other functions of the HOPSCA. In the Luozhuang District, Hedong District, the construction of commercial-oriented HOPSCA to reduce the flow of people into the old city. In the western city area, combined with the city village, suburban village transformation and trade city construction, build a number of various functions HOPSCA. 


\section{Planning and Design}

The third element of the HOPSCA development strategy is meticulous planning and design of commercial buildings. The planning and design of the HOPSCA focuses on local conditions, analysis of the location of the project market, economic and cultural conditions, to find a design plan in line with the situation of Linyi City program. Planning and design need to consider the correct planning layout, rational integration of design, improve the parking organization and transportation systems, interior open space design and so on. [3]

Specifically, the HOPSCA of Linyi city can improve the original urban environment, to re-shape the image of the old city center. In the building, HOPSCA in the old city center and the original elements of the old city echoes; in the architectural style, it is necessary to have innovation, but also in line with market conditions. In the traffic organization, to develop underground transportation and three-dimensional traffic. Linyi City core area or Beicheng New HOPSCA, relatively speaking, its planning is reasonable, the main focus on the establishment of functional systems for the city to increase the image of the highlights. In other central areas, the surrounding environment is better, less restrictive factors, in the architectural style and the choice of building materials, and strive to innovation, reflecting the city's new image. [4]

\section{Investment Operations}

The last strategy of HOPSCA development is the formulation and implementation of investment operation, which is the key to the development process. The main store investment can successfully achieve the desired results, a comprehensive investment stage can reach format, brand positioning with the optimal combination of positioning to determine whether the pre-positioning goals, but also to ensure the performance of the latter part of the operation and rental income win-win situation.

In today 's commercial real estate market in China, Wanda Group is the leader in this field, its third generation of Wanda Plaza has become synonymous with the Chinese HOPSCA. In the twenty-five years of development process, Wanda Group with its excellent corporate culture and scientific strategic planning and implementation, and always adhere to innovation, and actively build the core competitiveness of enterprises, so at this stage to build a successful HOPSCA operating model, for the industry to establish the benchmark.

The unique operation mode of Wanda HOPSCA is based on the external environment and the organization itself, and this model is fully researched from the academic level, which not only has positive effects on the development of HOPSCA and related enterprises, but also the government functions of the policy-making, industry planning to provide valuable inspiration. Moreover, in the historical background of actively promoting the new urbanization, to build a harmonious society is of great significance.

\section{Suggestions On PROMOTING THE CONSTRUCtion OF HOPSCA IN LINYI CITY}

HOPSCA is a product of urban economic development and social progress. It provides a more convenient, more functional and complete place for people's work, life, leisure, residence and so on, which has positive economic and social meanings while promoting the city image. Development and construction of a good HOPSCA, should pay attention to the following aspects:

- The policy aspects. Linyi HOPSCA construction is entering a rapid development stage. While recognizing the importance of HOPSCA in the development and operation of a city, the government should make full use of the scientific concept of development to avoid blind decision-making and blind construction. HOPSCA construction and operation cycle is long, involved in many administrative departments, need their co-operation.

- The development process. From location, positioning, planning and design to investment operations, in the HOPSCA throughout the development process, each stage must be repeated demonstration, careful confirmation, and strive to step by step. The risk of HOPSCA development is far greater than ordinary simple real estate projects. Regional economic environment is not in place, the industry market environment changes, project planning and positioning errors, funding strand breaks, developers operating capacity of the trader, the latter part of the operating disadvantage ....... and so on and so on almost every city has a HOPSCA failure cases . Therefore, it is necessary to prevent all kinds of risks in the process of HOPSCA construction, such as location risk, financial risk, planning design risk and market competition risk. [5]

\section{CONCLUSION}

Although the development of HOPSCA is in full swing, the research on HOPSCA is not much, and the focus of research is on planning and design and function orientation. The research area is mostly concentrated in a first and second-tier city. This study tries to study the development regularity and characteristics of HOPSCA in Linyi city from the perspective of urban development, and to explore the risk of HOPSCA development in Linyi city and explore the rationality of HOPSCA quantity and scale. At the same time, drawing lessons from the experience and lessons of the development of HOPSCA at home and abroad, put forward suggestions and suggestions for the future development of Linyi city HOPSCA.

\section{REFERENCES}

[1] Guan Jing, on the evolution of HOPSCA location and function in recent years - Taking Wanda Plaza in Nanchang as an example [J] .Jiangxi Chemical Industry, 2013 (4): 245-247.

[2] Huang Yejun, Ningbo HOPSCA development countermeasure research [J]. Economic Series, 2011 (3): 11-19. 
[3] He Jianguo, Zhao Cuicui, HOPSCA planning and design discuss [J]. Technology and Market, 2013 (7): 244.

[4] Gan Mengzhou, on the HOPSCA architectural design [J]. Urban architecture, 2013, (14): 21.

[5] Ma Zongguo, China's HOPSCA development approach [J]. Urban Development Research, 2011,18 (6): 14-16. 